\title{
Possible precursor to the March 11, 2011, Japan earthquake: ionospheric perturbations as seen by subionospheric very low frequency/ low frequency propagation
}

\author{
Masashi Hayakawa ${ }^{1,2,3,{ }^{\star}}$, Yasuhide Hobara ${ }^{2,4}$, Yoshihiro Yasuda ${ }^{3}$, Hiroki Yamaguchi ${ }^{5}$, Kenji Ohta $^{6}$, \\ Jun Izutsu $^{6}$, Tohru Nakamura ${ }^{7}$
}

\author{
${ }^{1}$ University of Electro-Communications, Advanced Wireless Communications Research Center, Tokyo, Japan \\ ${ }^{2}$ University of Electro-Communications, Research Station on Seismo-Electromagnetics, Tokyo, Japan \\ ${ }^{3}$ University of Electro-Communications, Hayakawa Institute of Seismo-Electromagnetics Co. Ltd., Tokyo, Japan \\ ${ }^{4}$ University of Electro-Communications, Graduate School of Informatics and Engineering, Tokyo, Japan \\ ${ }^{5}$ Earthquake Analysis Laboratory, Information Systems Inc., Minato-ku, Tokyo, Japan \\ ${ }^{6}$ Chubu University, Kasugai Aichi, Japan \\ ${ }^{7}$ Kochi University, Akebono Kochi, Japan
}

\section{Article history}

Received August 9, 2011; accepted September 29, 2012.

Subject classification:

Earthquake prediction, Ionospheric perturbation, VLF/LF propagation.

\begin{abstract}
This study reports on a possible very low frequency/low frequency $(V L F / L F)$ subionospheric precursor to a recent earthquake in Japan. As the epicenter of this large Japanese earthquake on March 11, 2011, was located just on the great-circle path from one of our VLF/LF network stations (Chofu) to the NLK US transmitter, we examined the propagation characteristics mainly associated with the signals from the NLK transmitter, as observed at three of the stations in Japan (Chofu, Kasugai and Kochi). On March 5 and 6, 2011, a remarkable anomaly was found on the path from NLK to Chofu, which is highly likely to have been a precursor to this earthquake. The anomaly in the night-time average amplitude at Chofu was characterized by a serious decrease in the signal that exceeded $-4 \sigma(\sigma$ : standard deviations). The anomaly was found on the same days on the other propagation paths (from NLK to both Kasugai and Kochi), although it was less enhanced. Finally, this propagation anomaly is extensively discussed with respect to the geomagnetic activity, and we also compare this anomaly with the properties related to the former 2004 Sumatra earthquake that had nearly the same magnitude as this March 11, 2011, earthquake.
\end{abstract}

\section{Introduction}

Very low frequency/low frequency (VLF/LF) subionospheric propagation data have recently been used extensively to monitor lower ionospheric perturbations associated with earthquakes [e.g., Hayakawa 2007, Hayakawa 2009, Hayakawa 2010, Chakrabarti 2010]. There have thus been a substantial number of studies published on such seismo-ionospheric perturbations. A recent study by
Hayakawa et al. [2010] established a statistically significant correlation of ionospheric perturbations detected as VLF/LF subionospheric propagation anomalies with earthquakes with large magnitudes $(>6.0)$ and shallow depths. This was a statistical study on the basis of long-term (seven years) data recorded in and around Japan. That study can be considered as further confirmation of our previous statistical studies [Gokhberg et al. 1989, Rozhnoi et al. 2004, Maekawa et al. 2006, Kasahara et al. 2008], which examined shorter periods of data, of the order of a few years.

Case studies of different large earthquakes are also of vital importance for investigations into the temporal and spatial characteristics of seismo-ionospheric perturbations. As was recently described in a review by Hayakawa [2009], these case studies include: (i) the Izu peninsula March 1997 earthquake swarm; (ii) the September 25, 2003, Tokachi-oki earthquake; (iii) the October 23, 2004, Niigata-chuetsu earthquake; (iv) the 1999 Chi-chi earthquake in Taiwan; and (v) the 2004 Sumatra earthquake; among others. The present study concerns the latest earthquake in Japan, on March 11, 2011.

\section{The 2011 Tohoku earthquake}

There was an extremely large earthquake (magnitude, 9.0) under the sea bed in the Pacific ocean off the Tohoku area of Japan, which is formally named as the 2011 earthquake off the Pacific coast of Tohoku. This earthquake took place at 14:46:18 LT on March 11, 2011, with its 


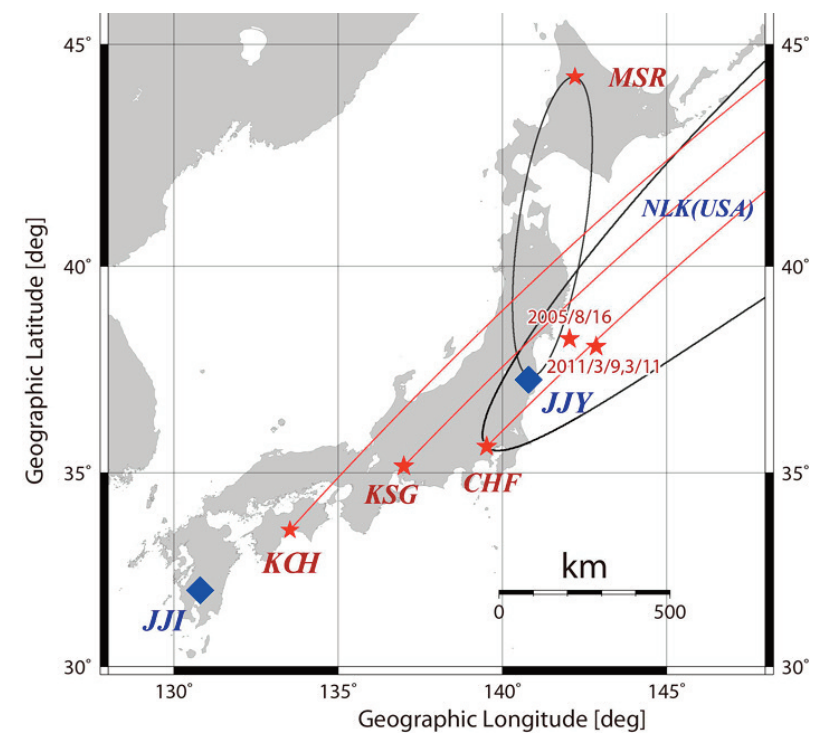

Figure 1. Relative locations of the JJY transmitter and the Moshiri (MSR) station, with the wave sensitive area. Also, the great-circle paths for the NLK (US transmitter) to Japanese receiving stations of Chofu (CHF), Kasugai (KSG) and Kochi $(\mathrm{KCH})$ are shown. The corresponding wave sensitive area is only shown for the NLK-CHF path. The epicenters of the three relevant earthquakes are shown: (i) the August 16, 2005, Miyagi-oki earthquake (2005/8/16); and (ii) the March 9, 2011, foreshock, and the March 11, 2011, main shock earthquakes (2011/3/9,3/11).

epicenter at the geographic coordinates $\left(36^{\circ} 6.2 \mathrm{~N}\right.$, $142^{\circ} 51.6 \mathrm{E}$ ), as shown in Figure 1, and with a depth of ca. 20 $\mathrm{km}$. This earthquake was a very typical oceanic earthquake of the plate type, which are very different from fault-type earthquakes, such as the Kobe earthquake, the Niigatachuetsu earthquake, and so it was of great concern.

\section{The Japanese VLF/LF network}

The Japanese and Pacific network for subionospheric VLF/LF propagation was established just after the 1995 Kobe earthquake, within the framework of the former NASDA frontier project (Hayakawa et al., 2004). The main observatories involved at present are: (1) Moshiri (MSR) in Hokkaido; (2) Chofu (CHF) and (3) Kasugai (KSG) near Nagoya; and (4) Kochi (KCH) and (5) Tsuyama (TYM), Okayama, as shown by the stars in Figure 1 (except for TYM). Some additional observatories are planned to be built shortly. At each station, we normally detect the signals from the two Japanese transmitters with call signs of JJY (in Fukushima; $40 \mathrm{kHz}$ ) and JJI (in Miyazaki, Kyusyu; $22.2 \mathrm{kHz}$ ), as shown by the diamonds in Figure 1. We can also detect the signals from a few foreign transmitters (e.g., NWC in Australia; NPM in Hawaii; and NLK in the US). The details of this VLF/LF network can be found in Hayakawa et al. [2004] and Hayakawa [2007, 2009, 2010].

\section{Observational results and analysis method}

Figure 1 illustrates the path from JJY to MSR, along with its corresponding 5th Fresnel zone, as the wave sensitive area, and the three paths from NLK (Seattle, USA) to the other Japanese observatories (CHF, KSG and $\mathrm{KCH}$ ). Furthermore, the 5th Fresnel zone for the propagation path from NLK to CHF is shown, which is the wave sensitive area for this path and which is much larger than that for the path from JJY to MSR, as the NLK-CHF propagation distance is much larger than that for the JJY-MSR path. These wave sensitive areas mean that any earthquakes that take place within the wave sensitive area can have a certain significant influence on the signals received at the observatory, which is termed a propagation anomaly (either in amplitude or in phase, or for both).

The analysis in this study follows the night-time fluctuation method [e.g., Hayakawa et al. 2010], which focuses only on the night-time amplitude data. We monitored the temporal evolution of amplitude $A(t)$ at a current time $t$ during the local night-time on a particular day, while $<\mathrm{A}(\mathrm{t})>$ was estimated as the average amplitude at the same time $t$ during the period from one day to 30 days before the current day. Then, we estimated the difference $d A(t)=$ $A(t)-<A(t)>$. Using this difference, we can estimate the most important parameter, the 'trend', as the night-time average amplitude (mean of the $\mathrm{dA}(\mathrm{t})$ during the local time). The second parameter is the dispersion, which is characterized by how much the amplitude fluctuates around the average, and the third parameter is the integration of $\mathrm{dA}(\mathrm{t})<0$ during the night, the night-time fluctuation. All of these parameters are normalized by their corresponding standard deviations over -30 days to -1 day before the current day. Further details can be found in Kasahara et al. [2008] and Hayakawa et al. [2010].

For the definition of the night-time period, we take the UT period of UT $=11 \mathrm{~h}-19 \mathrm{~h}$ for the propagation path from JJY to MSR. However, the definition of night-time is considerably complex for the east-west long-distance propagation from NLK to the Japanase stations (such as $\mathrm{CHF}$ ). By considering the sunrise and sunset both at the transmitter and at the receiving observatory (i.e., the terminator times) [Hayakawa et al. 1996] and also by checking the actual diurnal variations for the relevant NLKCHF path, we took UT $=9 \mathrm{~h}$ to $14 \mathrm{~h}$ for the night-time for the NLK-CHF path (i.e., the only period when the propagation path was completely in the dark).

\subsection{No precursor propagation anomaly for the JJY-MSR path}

As shown in Figure 1, a previous earthquake known as the 2005 Miyagi-oki earthquake (August 16, 2005; magnitude, 7.2) occurred very close to the wave sensitive area of the JJY-MSR propagation path (with the same magnitude as the foreshock on March 9, 2011). Here, we observed very significant precursor ionospheric perturbations on this propagation path [Muto et al. 2009].

However, the epicenter of this March 11, 2011, earthquake was shown to be located considerably far from the 

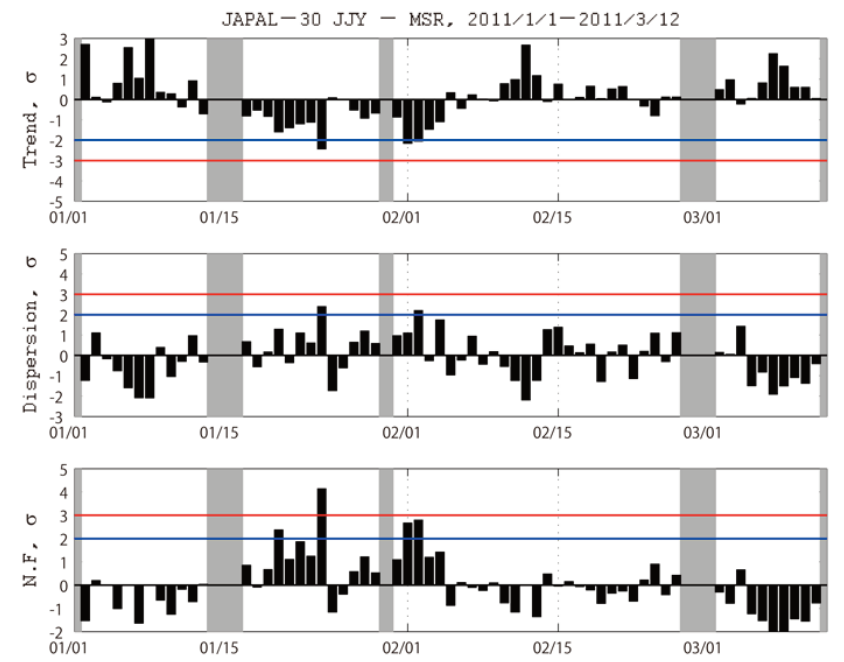

Figure 2. The temporal evolution of the propagation characteristics for the path of JJY-MSR during the period from January 1 to March 12, 2011. Pay attention only to the period from March 1 to March 12 to define any precursor to the March 11, 2011, earthquake. Top: trend (average night-time amplitude). Middle: dispersion (as a measure of the amplitude fluctuation). Bottom: night-time fluctuation, as defined by the night-time integration of the area of $\mathrm{dA}(\mathrm{t})<0$. The gray areas indicate periods with no observations.

wave sensitive area of the JJY-MSR path, as this earthquake occurred ca. $150 \mathrm{~km}$ away from the coast line. This might suggest that we would not expect any perturbation on the JJYMSR path for this earthquake. Figure 2 illustrates the temporal evolution of the propagation characteristics over the JJY-MSR path during the time period up to this March 11, 2011, earthquake. We pay attention only to the period from March 1 to March 9, so before the earthquake. In this period there was definitely no time interval in which the trend showed a decrease, together with the simultaneous increases in the dispersion and the night-time fluctuation parameters. Hayakawa et al. [2010] indicated that a propagation anomaly is characterized by a decrease in the trend, with simultaneous increases in the dispersion and the night-time fluctuation parameters. The experimental observation of no precursory anomaly appears to be very consistent with the initial theoretical expectation, as judged from the position of this earthquake relative to the propagation path.

4.2. Significant propagation anomalies associated with the propagation paths for the US transmitter NLK

Figure 1 suggests that the propagation paths from Japanese receiving stations (CHF, KSG and $\mathrm{KCH}$ ) to the US NLK transmitter are favorably located with respect to the epicenter of this March 11, 2011, earthquake. In particular, the NLK-CHF path passed just above the earthquake epicenter, and the corresponding wave sensitive area for this NLK-CHF path is shown as a dotted line. Two other propagation paths, from NLK to KSG and from NLK to KCH, were also favorable for us to note any corresponding ionospheric perturbations.

In response to these theoretical expectations, Figure $3 \mathrm{a}-\mathrm{c}$ illustrates the actual temporal evolutions of the propagation
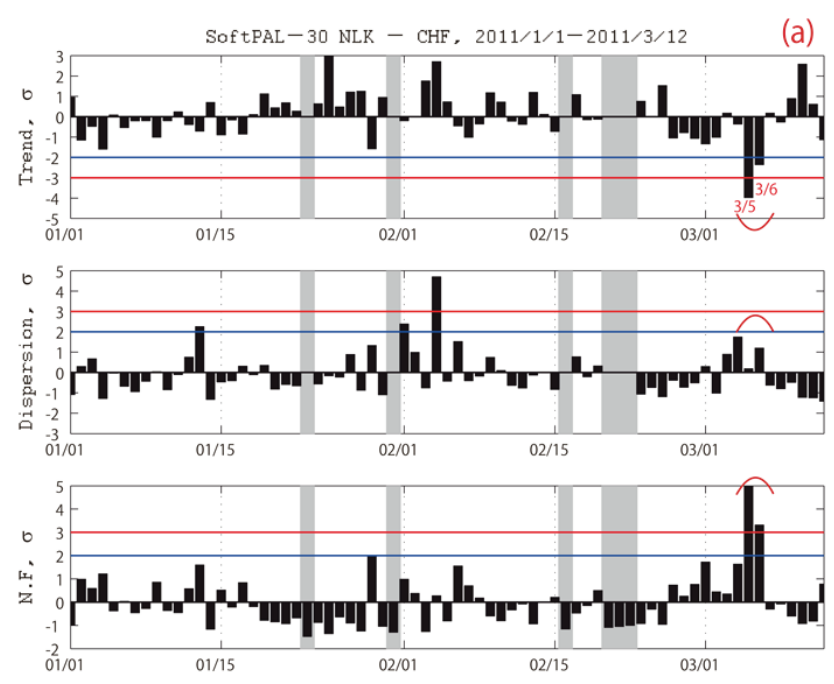

(b)
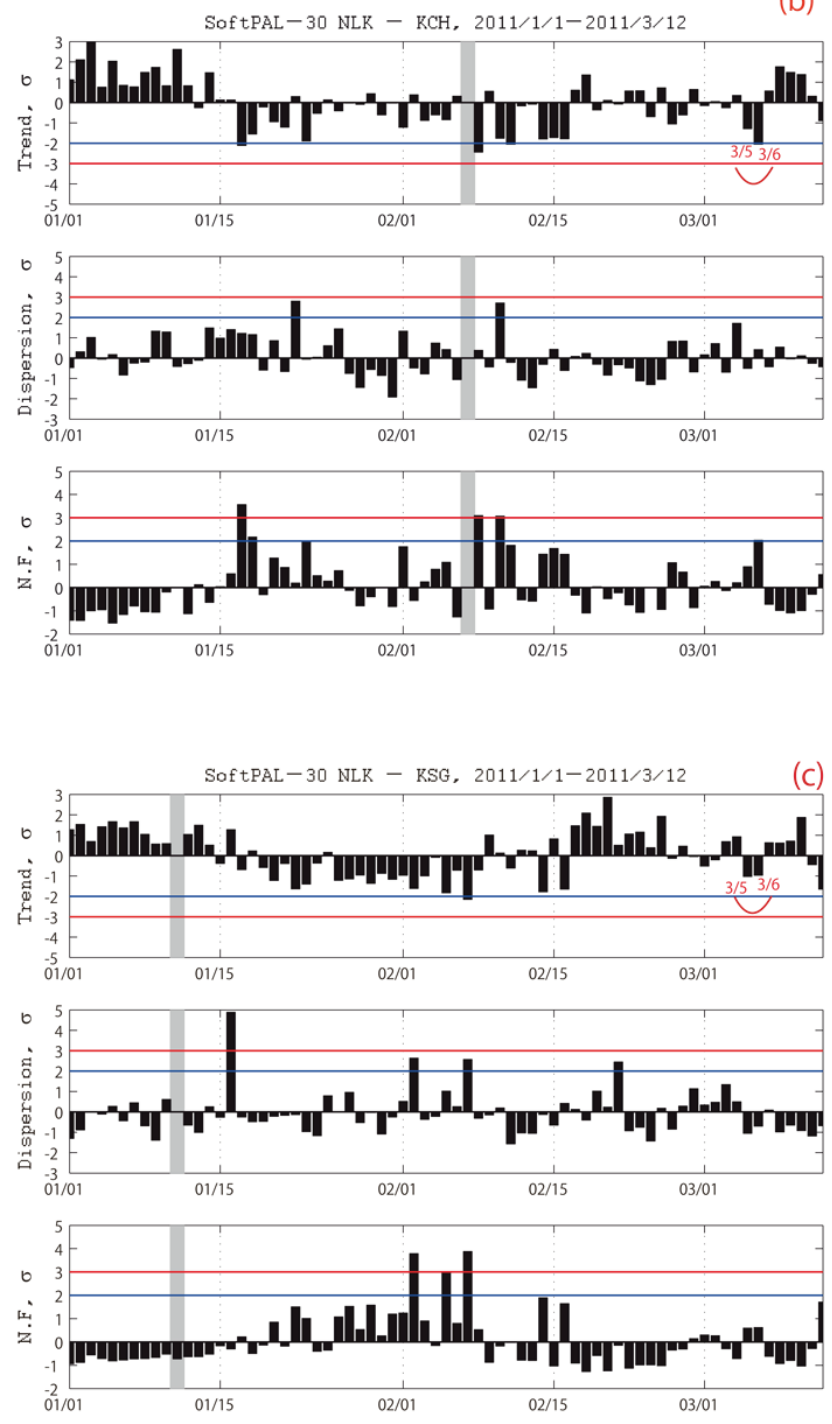

Figure 3. Temporal evolution of the propagation characteristics for the three propagation paths: (a) NLK-CHF; (b) NLK-KCH; and (c) NLK-KSG. The period from January 1 to March 12, 2011, is shown. Pay attention to the period from March 1 to March 12, to define any precursor to the March 11, 2011, earthquake. (a, b, c) Top: trend (average night-time amplitude). Middle: dispersion (as a measure of the amplitude fluctuation). Bottom: night-time fluctuation, as defined by the night-time integration of the area of $\mathrm{dA}(\mathrm{t})<0$. Any significant anomaly should be indicated in (a), (b) and (c). 


\section{$2011 \Sigma \mathrm{Kp}$}

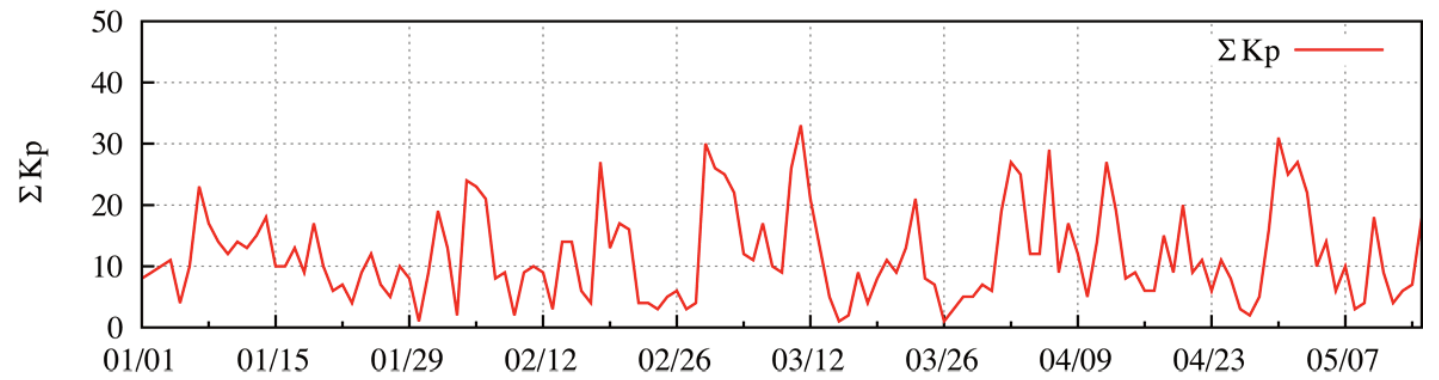

Figure 4. Temporal elevation of the geomagnetic activity ( Kp index) during the relevant period for the March 11, 2011, earthquake.

characteristics for these relevant paths; Figure 3a shows the NLK-CHF path, Figure 3b, the NLK-KCH path, and Figure $3 \mathrm{c}$, the NLK-KSG path. In each panel of Figure 3 the three physical parameters are shown: from top to the bottom, the trend, dispersion and night-time fluctuation, and these parameters are all normalized by their corresponding standard deviations $(\sigma)$. With the propagation path of NLK$\mathrm{CHF}$ in Figure 3a, we can pay attention to the period before the earthquake on March 11, 2011. We can clearly note a significant propagation anomaly on the two days of March 5 and 6,2011 . In particular, the propagation anomaly on March 5 is characterized by a remarkable decrease in the trend (exceeding $-3 \sigma$, or even more), together with simultaneous increases in the second parameter (dispersion) and in the night-time fluctuation. The corresponding anomaly can also be recognized in Figure $3 \mathrm{~b}$ for the propagation path of NLK-KSG. The anomaly for the path of NLK-KCH in Figure $3 \mathrm{~b}$ is particularly evident because the most important parameter, the trend, showed a significant decrease, reaching the $-2 \sigma$ level. On the other hand, the anomaly for the path of NLK-KSG in Figure $3 \mathrm{c}$ is less enhanced on the same days of March 5 and 6, 2011, although the response to this earthquake is very evident.

\section{Summary and discussion}

By making full use of our Japanase VLF/LF network, we have defined the following observational results for this large March 11, 2011, earthquake:

1. No definite anomaly was observed for the JJY-MSR path, as the earthquake epicenter was well away from the wave sensitive area of this propagation path.

2. There was a significant propagation anomaly on March 5 and 6 (about 5-6 days before the earthquake) especially for the NLK-CHF path. This anomaly is characterized by a remarkable decrease in the trend, together with clear enhancements in the dispersion and the night-time fluctuation. The same anomaly was also observed for the other two paths: NLK-KCH and NLK-KSG.

3. This earthquake was a consequence of an oceanic earthquake that was due to the movement of the plates, so that the result in the present study is the first report for any large plate-type earthquake in the sea.
We also discuss further the above summary. First of all, we ask whether the anomaly on March 5 and 6, 2011, was really a precursor to the March 11, 2011, earthquake. The most serious point for this problem is that the geomagnetic activity might have some influence on our summary. Figure 4 illustrates the temporal evolution of geomagnetic activity measured according to $\Sigma \mathrm{Kp}$ (the daily sum of the $\mathrm{Kp}$ index) during the relevant period, from late February to around March 10. As can be seen from Figure 4, the geomagnetic activity on March 5 and 6, 2011, when the anomaly was observed, was relatively quiet (of the order of $\Sigma \mathrm{Kp}=10-15$ ), although we note some enhancement of the geomagnetic activity in late February and around March 10. Thus, it is likely that the geomagnetic activity has nothing to do with this earthquake. As summarized as point (2) above, we found a significant propagation anomaly on the path of NLK-CHF. This means that the earthquake responsible for this anomaly might be located at any position along the path of NLK-CHF, as we have no propagation paths crossing these NLK-Japanese observatories. After having examined the earthquakes over the whole area of this propagation path, we were not able to find any earthquakes on the path during the propagation path. Thus, the propagation anomaly on March 5 and 6, 2011, shown in Figure 3 is very likely to be a precursor to the March 11, 2011, earthquake. The lead time for this earthquake was about 5-6 days, which appears to be consistent with the corresponding statistical results for land earthquakes in and around Japan reported by Hayakawa et al. [2010].

It is interesting for us to compare these characteristics of the ionospheric perturbations for this earthquake with those of an earthquake with nearly the same magnitude; i.e., the 2004 Sumatra earthquake with a magnitude of 9.1 [Horie et al. 2007a, b]. On the basis of the ground-based observations in Japan [Horie et al. 2007a, b] for the propagation paths associated with the Australian NWC transmitter, and also on the satellite observations of whistler-mode signals from the same transmitter [Molchanov et al. 2006], we found that the radius of the ionospheric perturbations was of the order of 2.5 $\mathrm{Mm}$ for the Sumatra earthquake. This earthquake was also an oceanic earthquake of the plate type, so the same type as the March 11, 2011, Tohoku earthquake. However, the most important difference between these two 
earthquakes is that the Sumatra earthquake occurred very close to the land area of Indonesia (so we would expecting a lot of influence on the land area), whereas the Tohoku earthquake occurred far from the coast line (ca. $150 \mathrm{~km}$ ), and thus right in the ocean. If we assume the same spatial scale for this March 11, 2011, earthquake as for the Sumatra earthquake, we would expect any significant perturbations to extend even to the path of JJY-MSR, but Figure 2 indicates that there was no anomaly at all on this path. This means that the spatial scale of ionospheric perturbations for the March 11, 2011, earthquake was considerably smaller, as compared with that for the 2004 Sumatra earthquake. Also, we can assume that this is probably closely related with the fact that the earthquake occurred completely in the sea, so that there are no such significant effects on the ionosphere due to the presence of the sea water.

\section{References}

Chakrabarti, S.K., ed. (2010). Propagation Effects of Very Low Frequency Radio Waves, AIP Conference Proceedings, vol. 1286, $362 \mathrm{pp}$.

Gokhberg, M.B., I.L. Gufeld, A.A. Rozhnoy, V.F. Marenko, V.F. Yampolsky and E.A. Ponomarev (1989). Study of seismic influence on the ionosphere by super long wave probing of the Earth-ionosphere waveguide, Phys. Earth Planet. Inter., 57, 64-67.

Hayakawa, M., O.A. Molchanov, T.Ondoh and E. Kawai (1996). The precursory signature effect of the Kobe earthquake on VLF subionospheric signals, J. Comm. Res. Lab., Tokyo, 43, 169-180.

Hayakawa, M., O.A. Molchanov and the NASDA/UEC team (2004). Summary report of the NASDA earthquake remote sensing frontier project, Phys. Chem. Earth, 29, 617-625.

Hayakawa, M. (2007). VLF/LF radio sounding of ionospheric perturbations associated with earthquakes, Sensors, 7, 1141-1151.

Hayakawa, M. (2009). Lower ionospheric perturbations associated with earthquakes, as detected by subionospheric VLF/LF radio waves, In: Electromagnetic Phenomena Associated with Earthquakes, M. Hayakawa (ed.), Transworld Research Network, Trivandrum (India), Chapter 5, 119-136.

Hayakawa, M (2010). The use of subionospheric VLF/LF propagation for the study of lower ionospheric perturbations associated with earthquakes, In: Propagation Effects of Very Low Frequency Radio Waves, S.K. Chakrabarti (ed.), Am. Inst. Physics, AIP Conference Proceedings, 1286, 223-269.

Hayakawa, M., Y. Kasahara, T. Nakamura, F. Muto, T. Horie, S. Maekawa, Y. Hobara, A.A. Rozhnoi, M. Solovieva and O.A. Molchanov (2010). A statistical study on the correlation between lower ionospheric perturbations as seen by subionospheric VLF/LF propagation and earthquakes,
J. Geophys. Res., 115, A09305; doi:10. 1029/2009JA015143.

Horie, T., S. Maekawa, T. Yamauchi and M. Hayakawa (2007a). A possible effect of ionospheric perturbations associated with the Sumatra earthquake, as revealed from subionospheric very-low-frequency (VLF) propagation (NWCJapan), Intl. J. Remote Sensing, 28, 3133-3139.

Horie, T., T. Yamauchi, M. Yoshida and M. Hayakawa (2007b). The wavelike structures of ionospheric perturbation associated with Sumatra earthquake of $26 \mathrm{De}-$ cember 2004, as revealed from VLF observation in Japan of NWC signals, J. Atmos. Solar-Terr. Phys., 69, 1021-1028.

Kasahara, Y., F. Muto, T. Horie, M. Yoshida, M. Hayakawa, K. Ohta, A. Rozhnoi, M. Solovieva and O. A. Molchanov (2008). On the statistical correlation between the ionospheric perturbations as detected by subionospheric $\mathrm{VLF} / \mathrm{LF}$ propagation anomalies and earthquakes, Nat. Haz. Earth System Sci., 8, 653-656.

Maekawa, S., T. Horie, T. Yamauchi, T. Sawaya, M. Ishikawa, M. Hayakawa and H. Sasaki (2006). A statistical study on the effect of earthquakes on the ionosphere, based on the subionospheric LF propagation data in Japan, Ann. Geophysicae, 24, 2219-2225.

Molchanov, O., A. Rozhnoi, M. Solovieva, O. Akentieva, J.J. Berthelier, M. Parrot, F. Lefeuvre, P.F. Biagi, L. Castellana and M. Hayakawa (2006), Global diagnostics of the ionospheric perturbations related to the seismic activity using the VLF radio signals collected on the DEMETER satellite, Nat. Haz. Earth System Sci., 6, 745-753.

Muto, F., T. Horie, M. Yoshida, M. Hayakawa, A. Rozhnoi, M. Solovieva and O.A. Molchanov (2009), Ionospheric perturbations related to the Miyagi-oki earthquake on $16 \mathrm{Au}$ gust 2005, as seen from Japanese VLF/LF subionospheric propagation network, Phys. Chem. Earth, 34, 449-455.

Rozhnoi, A., M.S. Solovieva, O.A. Molchanov and M. Hayakawa (2004). Middle latitude LF ( $40 \mathrm{kHz}$ ) phase variations associated with earthquakes for quiet and disturbed geomagnetic conditions, Phys. Chem. Earth, 29, 589-598.

\footnotetext{
${ }^{\star}$ Corresponding author: Masashi Hayakawa, Advanced Wireless Communications Research Center, University of Electro-Communications (UEC), Chofu, Tokyo, Japan; email: hayakawa@whistler.ee.uec.ac.jp.

(C) 2012 by the Istituto Nazionale di Geofisica e Vulcanologia. All rights reserved.
} 